\title{
Engineering Internal Control Analysis Based on AHP-FCE Taking Wind Power Enterprise as an Example
}

\author{
Lv Xiaoxiao ${ }^{1, a}$, Hou Zhengnan ${ }^{* 2, b}$,Tang Ming ${ }^{3}$,Zhuang Shengxian ${ }^{4}$ \\ ${ }^{1}$ School of Economics and Management Southwest Jiaotong University, Chengdu China \\ ${ }^{2}$ School of Electrical Engineering Southwest Jiaotong University, Chengdu China \\ 3 School of Economics and Management Southwest Jiaotong University, Chengdu China \\ 4 School of Electrical Engineering Southwest Jiaotong University, Chengdu China
}

\begin{abstract}
Wind power is a key development project. In recent years, the wind power industry has developed rapidly. The market needs enterprises with complete systems and higher work efficiency. Internal control is an important measure for the transparency and standardization of enterprise operations. Therefore, after implementing internal control on wind power companies, how to objectively evaluate the internal control construction of wind power companies has become a new research focus. This paper proposes a method to quantitatively evaluate the internal control of wind power enterprises. First, the internal control objectives are decomposed into multiple factor indicators, and then the internal control evaluation system of wind power enterprises is constructed, which is realized by the use of analytic hierarchy process (AHP) and fuzzy comprehensive evaluation (FCE). The calculation example shows that the quantitative evaluation method helps to find the deficiencies of the internal control of wind power enterprises, and provides a reference for the establishment and implementation of the system and the efficiency of business operation.
\end{abstract}

\section{Introduction}

Wind energy is a clean and renewable energy source, and one of the most promising power generation methods in the new energy development strategy ${ }^{[1]}$. Wind power is a key development project in the world. The wind power industry has developed rapidly in recent years. The development of the three leading manufacturers of wind power equipment manufacturing companies in China has been very stable in the industry. As of the end of 2016, Chinese cumulative wind power installed capacity accounted for more than $1 / 3$ of the world's total installed capacity, even for wind power ${ }^{[2]}$. The development of power generation is so rapid, but compared with traditional energy generation, wind power still has a lot of room for development, and wind power companies still have a long way to go.

Internal control is an important measure for the transparency and standardization of enterprise operations. In the golden stage of wind power development, enterprises with complete systems and higher work efficiency are more needed ${ }^{[3]}$. Therefore, after implementing internal control on wind power companies, how to objectively evaluate the internal control construction of wind power companies has become a new research focus.

At present, there are only directional guidelines for internal control evaluation, and there is no specific implementation method. This article defines the levels of internal control index division and the concept of indicators and conducts research on the causes of internal control risks, using fuzzy comprehensive evaluation (FCE) and levels Analytical method (AHP), a model for systematic quantitative evaluation of enterprise internal control is proposed to evaluate the internal control examples of wind power enterprises.

\section{AHP-FCE model}

\subsection{Analytic Hierarchy Process}

Analytical Hierarchy Process (AHP) refers to the use of tiered method to construct an evaluation factor index system. The factor indexes of each level are compared to obtain the relative importance degree, and the judgment matrix is established. By calculating the maximum value of the judgment matrix and its corresponding. The feature vector gets the weight value and finally the consistency test is used to verify the objectivity of the weight.

Relative importance refers to the degree of influence of $u_{i}$ factor and $u_{j}$ factor at the same level on the indicators $a_{i}$ and $a_{j}$ of the previous level. It can be expressed as $a_{i j}=a_{i} / a_{j}$. The widely-used nine-scale method is applicated, which is shown in Table 1.

\footnotetext{
axiaoxiaolv@my.swjtu.edu.cn

b* Corresponding author: zhengnanhou@my.swjtu.edu.cn
} 
Table1. The nine-scale method of relative importance

\begin{tabular}{|c|c|c|}
\hline $\mathbf{a}_{\mathbf{i j}}$ & Importance & Description \\
\hline 1 & Same important as $a_{j}$ & $\begin{array}{c}\mathrm{u}_{\mathrm{i}} \text { and } \mathrm{u}_{\mathrm{j}} \text { have the same impact on the indicators of the } \\
\text { previous level }\end{array}$ \\
\hline 3 & More important than $a_{j}$ & $\begin{array}{l}\mathrm{u}_{\mathrm{i}} \text { has a slightly impact on the indicators of the upper level than } \\
\qquad \mathrm{u}_{\mathrm{j}}\end{array}$ \\
\hline 5 & Obviously important than $\mathrm{a}_{\mathrm{j}}$ & $\mathrm{u}_{\mathrm{i}}$ has impact on the indicators of the upper level than $\mathrm{u}_{\mathrm{j}}$ \\
\hline 7 & Strongly important than $a_{j}$ & $\begin{array}{l}\mathrm{u}_{\mathrm{i}} \text { has a much greater impact on the indicators of the upper } \\
\qquad \text { level than } \mathrm{u}_{\mathrm{j}}\end{array}$ \\
\hline 9 & Absolutely important than $\mathrm{a}_{\mathrm{j}}$ & $\mathrm{u}_{\mathrm{i}}$ completely overwhelms $\mathrm{u}_{\mathrm{j}}$ on the upper level indicators \\
\hline $2 / 4 / 6 / 8$ & Between all levels & a compromise between two adjacent values \\
\hline reciprocal & Not important than $a_{j}$ & $\begin{array}{l}\mathrm{a}_{\mathrm{ij}}=1 / \mathrm{a}_{\mathrm{ji}} \text { Is the reciprocal of the relative importance of } \mathrm{u}_{\mathrm{j}} \\
\text { and } \mathrm{u}_{\mathrm{i}}\end{array}$ \\
\hline
\end{tabular}

According to the nine-scale method, each level of relative importance of $\mathrm{n}$ factors can establish the judgment matrix as

$$
\mathrm{C}=\left[\begin{array}{ccc}
\mathrm{a}_{11} & \cdots & \mathrm{a}_{1 \mathrm{n}} \\
\vdots & \ddots & \vdots \\
\mathrm{a}_{\mathrm{n} 1} & \cdots & \mathrm{a}_{\mathrm{nn}}
\end{array}\right]
$$

Solve the largest eigenvalue $\lambda_{\max }$ of the judgment matrix $\mathrm{C}$ and its corresponding largest eigenvector $\xi=$ $\left(\mathrm{x}_{1}, \mathrm{x}_{2}, \ldots, \mathrm{x}_{\mathrm{n}}\right)$. The weight of each factor can be obtained by normalizing $\xi$ to

$$
A=\left\{a_{1}, a_{2}, \ldots, a_{n}\right\}
$$

In this way, the weight of each factor in a single level is obtained. For a multi-layer system, from top to bottom, the weight of the upper-level factors can be calculated firstly, and then assigned to multiple sub-factors in the lower level, and finally the weight of the bottom-level factors to the overall goal can be obtained. If necessary, the factors can also be prioritized according to the weight.

Considering that the relative importance is artificially valued, the consistency test of the judgment matrix $\mathrm{C}$ should also be performed. Check the proportion of agreement

$$
\mathrm{C}_{\mathrm{R}}=\mathrm{C}_{\mathrm{I}} / \mathrm{R}_{\mathrm{I}}
$$

Among them, the consistency index $C_{I}=\frac{\lambda_{\max }-n}{n-1}$ and the average random consistency index $R_{I}$ can be obtained by looking up the table. If $C_{R}<0.1$ is satisfied, it can be considered that the judgment matrix is consistent, and the value of the relative importance is reasonable. Otherwise, the value of the judgment matrix needs to be adjusted to meet the consistency requirement. It is worth mentioning that the judgment matrix with only two factors already has complete consistency, and there is no need for consistency testing.

\subsection{Fuzzy Comprehensive Evaluation}

After using AHP to assign weights to each sub-factor index of the evaluation, it is necessary to evaluate each sub-factor index separately. However, for the internal control of public institutions, most of the factor indexes are qualitative (such as good or bad). In order to achieve quantitative evaluation, Fuzzy Comprehensive Evaluation (FCE) could be used.

Assuming that there are $\mathrm{m}$ evaluation levels for each factor index in the factor index set $U=\left\{\mathrm{u}_{1}, \mathrm{u}_{2}, \ldots, \mathrm{u}_{\mathrm{n}}\right\}$, the evaluation set can be expressed as $V=\left\{\mathrm{v}_{1}, \mathrm{v}_{2}, \ldots, \mathrm{v}_{\mathrm{m}}\right\}$ FCE regards the evaluation of any factor index $u_{i}$ as a mapping to the evaluation set $\mathrm{V}$, that is, the degree of membership of the evaluation $r_{i}=\left\{r_{i 1}, r_{i 2}, \ldots, r_{i m}\right\}$, where is the degree $r_{i j}$ of membership of the factor index $u_{i}$ to the evaluation $v_{j}$. Then the fuzzy evaluation matrix of the whole factor index of the whole evaluation system can be obtained as

$$
\mathrm{R}=\left[\begin{array}{ccc}
\mathrm{r}_{11} & \cdots & \mathrm{r}_{1 \mathrm{~m}} \\
\vdots & \ddots & \vdots \\
\mathrm{r}_{\mathrm{n} 1} & \cdots & \mathrm{r}_{\mathrm{nm}}
\end{array}\right]
$$

\subsection{Evaluation Model}

Considering that the final quantitative score is to be achieved, the evaluation set $\mathrm{V}$ can be expressed in the form of scores. For example, the three-segment \{good, medium, bad $\}$ can be expressed as $\{100,50,0\}$, that is to say, $\mathrm{v}_{1}, \mathrm{v}_{2}, \ldots \mathrm{v}_{\mathrm{m}}$ are quantified in the form of scores, then the evaluation set $\mathrm{V}$ can also be regarded as a digital matrix. Synthesizing the fuzzy evaluation matrix $R$ and the evaluation set matrix $\mathrm{V}$, the individual score of each element index can be obtained

$\mathrm{B}=\mathrm{R} \cdot \mathrm{V}^{\mathrm{T}}$

Using the weight $\mathrm{A}$ of each factor index obtained by AHP to weight the individual score B of each element index, the quantitative evaluation model of the internal control of public institutions can be obtained as

$$
\mathrm{E}=\mathrm{A} \cdot \mathrm{B}=\mathrm{A} \cdot \mathrm{R} \cdot \mathrm{V}^{\mathrm{T}}
$$

\section{Internal control of wind power companies}

\subsection{Evaluation system construction}

Internal control indicators have specific meanings at two levels. One is the relevant meaning contained in the establishment of the unit, and the other is the relevant meaning of business operation. The unit level focuses on the organization and construction of internal control work. The business level evaluates budget management, revenue and expenditure management, and procurement 
management. The realization of goals needs to rely on the combination of unit level and business level, and also needs to ensure effectiveness of design and the strength of implementation in business process control. The first task is to deconstruct the goal of internal control evaluation of the enterprise.

From the perspective of the unit level as a whole, the internal control evaluation first needs to cut in from the unit's power distribution and system completeness, and supervise these key points from a macro perspective. The unit level inspection in this article mainly involves the following modules: internal control work starts circumstances, the responsibility mechanism of the person in charge of the unit, the restriction mechanism of rights operation, the completeness of the system, the responsibilities of incompatible positions, and the application of the management information system.

When considering the business level, it is necessary to start with the main business chain of the unit, which is the risk control system of the business process in the daily economic activities of the unit. This article sets the internal control evaluation at the business level into six aspects of examination content, namely budget business, revenue and expenditure business, procurement business, assets, construction projects, and contracts.

Based on the above analysis, and at the same time by investigating the $\mathrm{X}$ wind power enterprise, the internal control evaluation index system established including: 2 B-level indicators, $12 \mathrm{C}$-level indicators and calculated according to the AHP-FCE model. The weight of each indicator is shown in Figure 1.

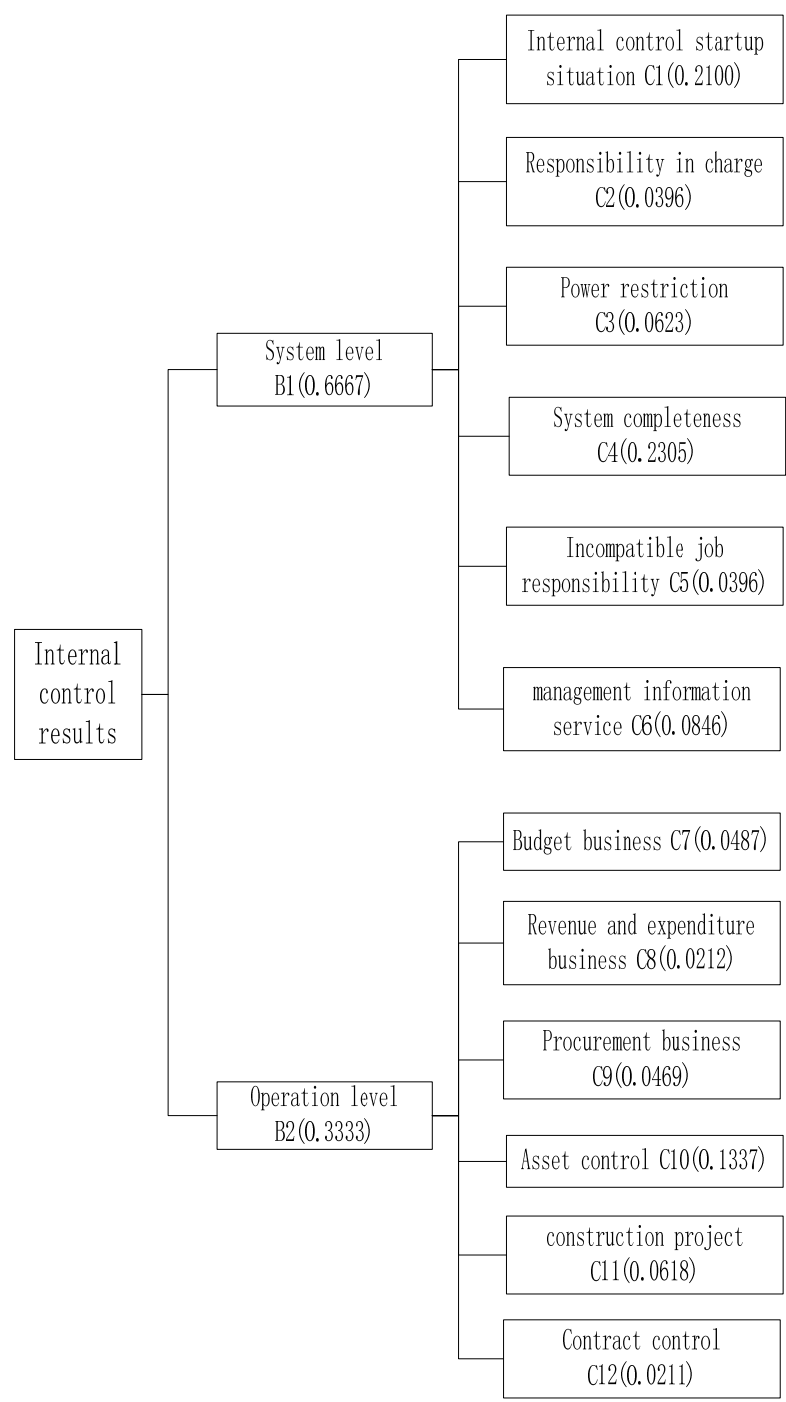

Figure 1. Internal control evaluation system

\subsection{Evaluation Results and Analysis}

For the internal control evaluation system of wind power enterprises proposed above, a judgment matrix of each element index is formed, and the weight of each element index of each layer is calculated as indicated in Figure 1. Comprehensively considering the fuzzy evaluation matrix and evaluation set, the comprehensive score of $\mathrm{X}$ Wind Power Company can be obtained as shown in Figure 2. 


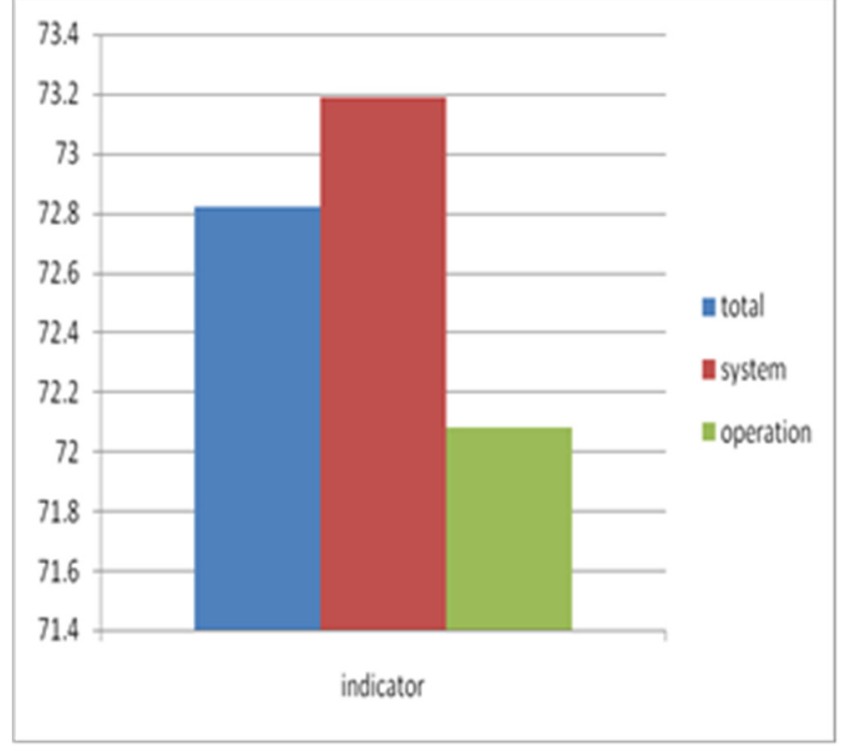

Figure 2. Comprehensive Score of X Wind Power Company

From the overall point of view, the company's internal control work has been implemented well. At the unit level, it has carried out relatively effective work including starting internal control work, reorganizing organizational structure, remedying system loopholes, and monitoring key position risks. At the business level, the system and implementation procedures for most of the business activities at the business level are clarified as a whole, but the system details fail to meet the standards of the internal control guidelines. There is a lack of corresponding control procedures in the specific implementation links, and the daily business operations lack uniformity and accountability. The system is not very clear, and these problems need to be solved urgently.

\section{CONCLUSION}

This paper uses the analytic hierarchy process and fuzzy comprehensive evaluation to put forward a method of quantitatively evaluating the internal control of wind power companies. Firstly, the key points of the internal control evaluation of wind power enterprises are deconstructed, the evaluation system is constructed, and the weight of each element index is obtained by AHP-FCE and the fuzzy evaluation matrix is formed, as well as the quantitative evaluation mathematical model of unit internal control. The calculation examples show that the method in this paper can comprehensively and quantitatively evaluate the internal control quality of wind power enterprises, help to discover potential problems and deficiencies of the enterprise system, and provide a reference for improving the efficiency of the enterprise and improving the internal control work.

\section{Acknowledgment}

This research was supported by the National Key R\&D Program of China (Grant No. 2017YFB1200703).

\section{References}

1. XU Jin, JIN Yi, HU Congchuan, et al. Research oncombined power transmission scheme for offshore windfarm cluster[J]. Power System and Clean Energy, 2016, 32(11): 107-113.

2. KHALID M, AGUILERA R P, SAVKIN A V, etal. On maximizing profit of wind-battery supported power station based on wind power and energy price forecasting[J]. Applied Energy, 2018, 211: 764-773.

3. XIA S W, CHAN K.W., LUO X, et al. Optimalsizing of energy storage system and its cost-benefit analysis for power grid planning with intermittent wind generation[J]. Renewable Energy, 2018, 122: 472486.

4. Peter Enevoldsen, Scott Victor Valentine, Benjamin Sovacool.Insights into wind sites: Critically assessing the innovation, cost, and performance dynamics of global wind energy development[J].Energy Policy, 2018, (120): 1-7.

5. Benjamin Sovacoolab, Peter Enevoldsen.One style to build them all: Corporate culture and innovation in the offshore wind industry[J].Energy Policy, 2015, (86): 402-415.

6. Bjarne Steffen.The importance of project finance for renewable energy projects[J].Energy Economics, 2018, (69): 280-294. 